\title{
An Energy-Saving Control Strategy with Load Sensing for Electro-Hydraulic Servo Systems
}

\author{
Weiping Wang $1,2,{ }^{*}-$ Bo Wang 1 \\ ${ }^{1}$ School of Mechatronic Engineering, China University of Mining and Technology, China \\ 2 Jiangsu Key Laboratory of Mine Mechanical and Electrical Equipment, China University of Mining and Technology, China
}

The electro-hydraulic servo system (EHSS) usually demonstrates a lower efficiency in comparison to other available actuation methods. Thus, an energy-saving control strategy based on a load-sensing structure is developed in this study to improve the system efficiency of EHSS. The presented controller employs variable supply pressure control (VSPC) to reduce the pressure loss across the valve. Unlike the existing VSPC, a load-sensing structure that consists of a load-sensing pump, a proportional relief valve (PRV), and a throttle valve is proposed. Therefore, the overflow loss can also be decreased. Additionally, an adaptive backstepping sliding mode control is presented for the purpose of position tracking. Compared with the fixed displacement system, the proposed method is capable of saving $62.5 \%$ and $90 \%$ energy for the harmonic reference tests and multi-step reference tests, respectively, during the task of position tracking.

Keywords: energy saving, position tracking, load sensing, electro-hydraulic

Highlights

- A load-sensing structure for improving the efficiency of electro-hydraulic servo systems is proposed.

- A variable supply pressure control strategy used for energy-saving is derived.

- $\quad$ An adaptive backstepping sliding mode control is designed for position tracking.

- Energy-saving performance and position tracking performance are both demonstrated.

\section{INTRODUCTION}

The electro-hydraulic servo system (EHSS) has been demonstrated to be a promising choice for diverse applications due to its high-power density, ease of implementation, good dynamic performance and durability, despite the rapid development of electric power transmission. Owing to these characteristic, the EHSS has been widely used in the aircraft control, machine tools and manufacturing, excavating, and automotive industries [1] to [4].

The problem of EHSS position tracking control has been addressed in many papers and numerous methods have been proposed to improve the position tracking control performance of the EHSS. To be specific, in [5], an extended disturbance observer is presented to achieve the position-tracking task for its fast response speed in handling the disturbance. An adaptive robust controller (ARC) has also been utilized for the trajectory tracking of hydraulic actuators in the presence of uncertain nonlinearities and parameter uncertainties [6] and [7]. A feedforward plus Linear Quadratic Regulator (LQR) controller and quantitative feedback controller (QFT) were proposed in [8] and [9], respectively, to improve the robustness of the EHSS. A compound control strategy based on velocity compensation and active disturbance rejection control was proposed in [10] to enhance the system's anti-jamming capability while simultaneously improving the control precision of the system. Although the aforementioned methods have been demonstrated to be successful for their specific purpose, the EHSS is not among the most efficient actuation methods from an energy-saving perspective, which may limit its further applications in industrial and machinery contexts.

Therefore, certain efforts have been made in the literature to improve the overall efficiency of the EHSS. An integrated control of clamping force and energy saving by using a load-sensing variable pump was proposed in [11]. The electrical adaptations of this method, electro-hydraulic load sensing (EH-LS), which includes a constant displacement pump and an $\mathrm{AC}$ motor, was also utilized to improve the energy efficiency of the EHSS [12] and [13]. However, the pressure difference across the valve, which is usually set at a constant value, is not optimized from an energy-saving perspective. Thus, the efficiency of the load-sensing pump could be further improved by reducing the pressure loss across the valve [14]. As an alternative, energy regeneration is also an attractive research field for energy saving of EHSS [15] and [16]. Specifically, in [16], the independent valve metering control method for a hydraulic manipulator using five cartridge valves and one accumulator was proposed. However, although a significant energysaving capability was obtained, the energy loss in the cartridge valve is still considerable.

It is clear that although a certain amount of energy can be saved by the aforementioned methods, system 
efficiency can be further improved by reducing the pressure loss across the valve. To achieve this goal, the variable supply pressure control (VSPC) has gained more attention in recent years. A switched linear control algorithm-based VSPC is proposed to improve the energy-saving capabilities of the EHSS in [4]. Another VSPC combined with the nonlinear backstepping controller which is presented in [3] also has an energy-saving effect on the EHSS. However, since the power unit in these methods is a constant displacement pump, the overflow loss still exists in these studies. In most cases, the maximum flow rate of the pump is much larger than the demand flow rate for a given trajectory. Thus, a large amount of energy will be consumed.

In this study, a load-sensing structure is proposed to reduce the overflow loss. Based on this structure, the VSPC is employed to decrease the pressure loss across the valve. Therefore, the flow and pressure of the system can both adapt to the control demand. Additionally, an adaptive backstepping sliding mode control is presented to achieve a precise position tracking performance. The dynamic behavior of the system and the effectiveness of the controller are verified with experiments.

\section{PROBLEM FORMULATION}

The schematic diagram of the proposed system is shown in Fig. 1. A proportional relief valve (PRV) is used in parallel with the hydraulic line at the location of the load-sensing pump to regulate the desired supply pressure. A proportional directional valve (PDV) is utilized to control the position of the cylinder.

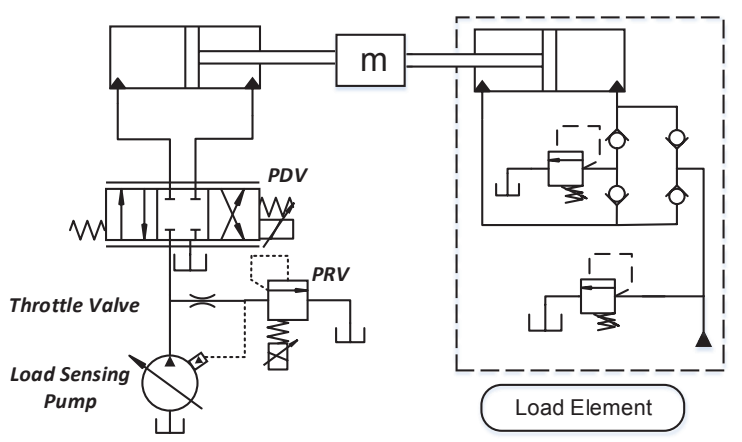

Fig. 1. Schematic diagram of the proposed electro-hydraulic system

Since the natural frequency of the servo valve used here is much higher than that of a typical EHSS, the valve dynamics are often neglected without a significant reduction in the control performance [5]. Therefore, the flow equation of the hydraulic valve can be written as:

$$
\left\{\begin{array}{c}
Q_{1}=k_{\mathrm{q}} k_{\mathrm{x}} \underbrace{\left[s_{\mathrm{g}}\left(u_{\mathrm{d}}\right) \sqrt{\frac{2}{\rho}\left(p_{\mathrm{s}}-p_{1}\right)}+s_{\mathrm{g}}\left(-u_{\mathrm{d}}\right) \sqrt{\frac{2}{\rho}\left(p_{1}-p_{\mathrm{t}}\right)}\right]}_{g_{1}} u_{\mathrm{d}} \\
Q_{2}=k_{\mathrm{q}} k_{\mathrm{x}} \underbrace{\left[s_{\mathrm{g}}\left(u_{\mathrm{d}}\right) \sqrt{\frac{2}{\rho}\left(p_{2}-p_{\mathrm{t}}\right)}+s_{\mathrm{g}}\left(-u_{\mathrm{d}}\right) \sqrt{\frac{2}{\rho}\left(p_{\mathrm{s}}-p_{2}\right)}\right]}_{g_{2}} u_{\mathrm{d}} \\
s_{\mathrm{g}}(\bullet)=\left\{\begin{array}{l}
1, \text { if } \bullet \geq 0 \\
0, \text { if } \bullet<0
\end{array},\right.
\end{array}\right.
$$

where is the flow gain coefficient of the servo valve, $k_{\mathrm{x}}$ is a positive constant, $p_{\mathrm{s}}$ is the supply pressure of the pump, $p_{\mathrm{t}}$ is the tank pressure, $\rho$ is the oil density, $p_{1}$ and $p_{2}$ are the chamber pressures of the cylinder, $Q_{1}$ is the supply flow rate to the forward chamber and $Q_{2}$ is the return flow rate from the return chamber, $u_{\mathrm{d}}$ is the input signal of PDV.

The pressure dynamics of the actuator can be written as:

$$
\left\{\begin{array}{l}
\dot{p}_{1}=h_{1}\left[Q_{1}-A_{1} \dot{x}_{\mathrm{p}}-C_{\mathrm{t}} p_{1}+C_{\mathrm{i}} p_{2}\right] \\
\dot{p}_{2}=h_{2}\left[-Q_{2}+A_{2} \dot{x}_{\mathrm{p}}-C_{\mathrm{t}} p_{2}+C_{\mathrm{i}} p_{1}\right]
\end{array}\right.
$$

where $x_{\mathrm{p}}$ is the piston position, $C_{\mathrm{t}}=C_{\mathrm{i}}+C_{\mathrm{e}}$ is the total leakage coefficient [17], $C_{\mathrm{e}}$ is the external leakage coefficient, $C_{\mathrm{i}}$ is the internal leakage coefficient, $h_{1}=\beta_{\mathrm{e}} / V_{1}, h_{2}=\beta_{\mathrm{e}} / V_{2}, V_{1}, V_{2}$ are the control volumes of the two chambers, respectively. $\beta_{\mathrm{e}}$ is the effective bulk modulus of the system.

The force balance equation of the system is expressed as:

$$
m \ddot{x}_{\mathrm{p}}=p_{1} A_{1}-p_{2} A_{2}-B_{\mathrm{c}} \dot{x}_{\mathrm{p}}-F_{\mathrm{L}}-F_{\mathrm{f}},
$$

where $m$ is the equivalent mass of the load, $x_{\mathrm{p}}$ is the displacement of the cylinder rod, $B_{\mathrm{c}}$ is the coefficient of viscous damping, $F_{\mathrm{L}}$ is the external load force, $F_{\mathrm{f}}$ is the Coulomb friction force.

Define the system state variables as Eq. (5):

$$
\left[x_{1}, x_{2}, x_{3}, x_{4}\right]^{\mathrm{T}}=\left[x_{\mathrm{p}}, \dot{x}_{\mathrm{p}}, p_{1}, p_{2}\right]^{\mathrm{T}} \text {. }
$$

In order to make the system fall into the strict feedback form, the state variables are reconstructed as Eq. (6), where $a=A_{2} / A_{1}$.

$$
\left[x_{1}, x_{2}, \bar{x}_{3}\right]^{\mathrm{T}}=\left[x_{\mathrm{p}}, \dot{x}_{\mathrm{p}}, p_{1}-a p_{2}\right]^{\mathrm{T}} \text {. }
$$

Considering the uncertainties and disturbance in the model, the entire system can be expressed in a state space form as Eq. (7): 


$$
\begin{aligned}
& \left\{\begin{array}{l}
\dot{x}_{1}=x_{2} \\
\dot{x}_{2}=\frac{A_{1}}{m} \bar{x}_{3}-\frac{B_{\mathrm{c}}}{m} x_{2}-\frac{F_{\mathrm{L}}}{m} \underbrace{F_{\mathrm{f}}}_{\Delta_{1}}+d_{1} \\
\dot{\bar{x}}_{3}=-f_{1} x_{2}-f_{2} x_{3}+f_{3} x_{4}+f_{4} u_{\mathrm{d}}+\underbrace{d_{2}}_{\Delta_{2}} \\
y=x_{1}
\end{array},\right. \\
& \left\{\begin{array}{l}
f_{1}=h_{1} A_{1}+h_{2} A_{2} a \\
f_{2}=h_{1} C_{\mathrm{t}}+h_{2} C_{\mathrm{i}} a \\
f_{3}=h_{1} C_{\mathrm{i}}+h_{2} C_{\mathrm{t}} a \\
f_{4}=h_{1} k_{\mathrm{q}} k_{\mathrm{x}} g_{1}+h_{2} k_{\mathrm{q}} k_{\mathrm{x}} g_{2} a
\end{array},\right.
\end{aligned}
$$

where $f_{1} \sim f_{4}$ are shown in Eq. (8) and $d_{1}, d_{2}$ represent the lumped modelling error including unmodelled dynamics and external disturbance. It can be seen that the system is highly nonlinear because it suffers from the lumped modelling error and parametric uncertainties.

The control task is summarized as follows: given the desired motion trajectory $x_{\mathrm{d}}$, the objective is to synthesize a control input of the PDV such that the output $x_{1}$ tracks $x_{\mathrm{d}}$ as closely as possible. Meanwhile, The PRV is utilized to regulate the supply pressure $p_{\mathrm{s}}$ in order to reduce the energy consumption.

\section{ENERGY-SAVING STRATEGY}

For a fixed displacement pump, there always exists excess pressure and overflow loss. The VSPC can reduce the excess pressure loss of the system. However, the overflow loss is not considered in these studies. The load-sensing pump can adjust the flow and pressure per the demand. Thus, the overflow loss can be eliminated by employing a load-sensing pump. However, the pressure margin of the loadsensing pump, usually fixed approximately 14 bar to 30 bar across the valve, is not optimized in studies involving load-sensing pumps. Thus, if the VSPC could be applied to the load-sensing pump, the system efficiency would be significantly improved.

\subsection{Configuration of the Load-Sensing Structure}

The load-sensing structure of the proposed system is shown in Fig. 2. The structure comprises the loadsensing pump, the PRV, and the throttle valve. If the pump pressure is larger than the sum of the preset pressure margin $p_{\mathrm{m}}$ and feedback pressure $p_{\mathrm{Ls}}$, the cylinder will be driven to reduce the angle of the swash plate $\theta$ until the pressure is balanced on both sides and vice versa. The pressure margin $p_{\mathrm{m}}$ is preset by the spring inside the load-sensing pump. However, this margin can be indirectly regulated by adjusting the feedback pressure $p_{\mathrm{Ls}}$, which is controlled by the PRV. The function of the throttle valve is only to establish the pressure difference between the pump and the PRV. The power consumed in the throttle valve is neglected because the orifice of the throttle valve can be adjusted to a small value and the pressure difference across the valve is equal to $p_{\mathrm{m}}$ when the system is stable.

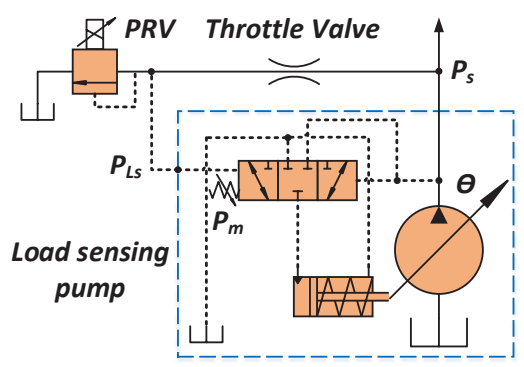

Fig. 2. Schematic diagram of load sensing

\subsection{Variable Supply Pressure Control}

For a certain demand flow, the smallest possible pressure drop will occur if the valve is fully opened. However, in that case, the PDV cannot be adjusted in a certain range to compensate for the tracking error. It can be concluded that if the opening of the PDV remains at a relatively high level during the forward and backward motion while the pump only provides the demand pressure, the pressure loss across the valve will be minimized. The VSPC is introduced as follows.

For the forward motion $\left(\dot{x}_{\mathrm{d}} \geq 0\right)$, the desired inlet pressure drop across the PDV $\Delta p_{1}=p_{\mathrm{s}}-p_{1}$ can be calculated as follows:

$$
\begin{gathered}
A_{1} \dot{x}_{\mathrm{d}}=k_{\mathrm{q}} k_{\mathrm{x}} \alpha u_{\text {max }} \sqrt{\frac{2}{\rho} \Delta p_{1}}, \\
\Delta p_{1}=\frac{\rho A_{1}^{2} \dot{x}_{\mathrm{d}}^{2}}{2 k_{\mathrm{q}}^{2} k_{\mathrm{x}}^{2} \alpha^{2} u_{\text {max }}^{2}},
\end{gathered}
$$

where $u_{\max }$ is the maximum input voltage of PDV during the forward motion, $\alpha$ is the desired normalized input signal of PDV determined by the users/designers to indicate the importance of energy saving. The value of $\alpha$ can adjust the desired spool position of the PDV, and a higher value of $\alpha$ can improve the energy efficiency.

The pressure drop between pump and valve can be obtained as: 


$$
\Delta p_{2}=\lambda \frac{l}{d} \frac{\rho \dot{x}_{\mathrm{d}}^{2}}{2},
$$

where $\lambda$ is the layer resistance coefficient, $l$ is the pipe length, $d$ is the pipe diameter.

Because of uncertainties in the plant, the design of the desired pump pressure must allow for a margin of safety. Thus, $\sigma_{1} p_{1}$ is added to the value of desired pump pressure [3]. From Eqs. (9), (10) and (11), the desired pump pressure, the indirect feedback pressure and the input signal of PRV can be written as:

$$
\begin{gathered}
p_{\mathrm{sd} 1}=p_{1}+\frac{\rho A_{1}^{2} \dot{x}_{\mathrm{d}}^{2}}{2 k_{\mathrm{q}}^{2} k_{\mathrm{x}}^{2} \alpha^{2} u_{\text {max }}^{2}}+\lambda \frac{l}{d} \frac{\rho \dot{x}_{\mathrm{d}}^{2}}{2}+\sigma_{1} p_{1}, \\
p_{\mathrm{Ls} 1}=u_{\mathrm{rv} 1} k_{\mathrm{rv}}=p_{\mathrm{sd} 1}-p_{\mathrm{m}} \rightarrow u_{\mathrm{rv} 1}=\frac{p_{\mathrm{sd} 1}-p_{\mathrm{m}}}{k_{\mathrm{rv}}},
\end{gathered}
$$

where $k_{\mathrm{rv}}$ is the PRV gain, $u_{\mathrm{rv} 1}$ is the control input of the PRV, $p_{\mathrm{sd} 1}$ is the desired pump pressure in the forward motion, $p_{\mathrm{Ls} 1}$ is the indirect feedback pressure governed by the PRV in the forward motion. Because the supply pressure always exceeds the cracking pressure, the dynamic of the PRV can be simplified as Eq. (13) [3].

As with the case of forward motion, the desired pump pressure, and control voltage of the PRV when $\dot{x}_{\mathrm{d}}<0$ are expressed as:

$$
\begin{aligned}
& p_{\mathrm{sd} 2}=p_{2}+\frac{\rho A_{2}^{2} \dot{x}_{\mathrm{d}}^{2}}{2 k_{\mathrm{q}}^{2} k_{\mathrm{x}}^{2} \alpha^{2} u_{\mathrm{max}}^{2}}+\lambda \frac{l}{d} \frac{\rho \dot{x}_{\mathrm{d}}^{2}}{2}+\sigma_{2} p_{2}, \\
& p_{\mathrm{Ls} 2}=u_{\mathrm{rv} 2} k_{\mathrm{rv}}=p_{\mathrm{sd} 2}-p_{\mathrm{m}} \rightarrow u_{\mathrm{rv} 2}=\frac{p_{\mathrm{sd} 2}-p_{\mathrm{m}}}{k_{\mathrm{rv}}} .
\end{aligned}
$$

Remark 1: It should be noted that the pump pressure will remain as the preset pressure margin $p_{\mathrm{m}}$ when the desired pump pressure is less than the $p_{\mathrm{m}}$. Furthermore, although the pump pressure is not zero when $\dot{x}_{\mathrm{d}}=0$, the input signal of PDV varies slightly around zero. Consequently, the pump only provides a little flow mainly for compensating for the internal leakage. Therefore, the energy consumption, in this case, is also very small.

\section{NONLINEAR POSITION TRACKING CONTROL}

To implement the proposed position tracking strategy, the following assumption should be made as follows.

Assumptions: $\theta_{1}, \theta_{2}$ are used to denote the dominant uncertain parameters $F_{\mathrm{L}} / m, B_{\mathrm{c}} / m$. The extent of parametric uncertainties and uncertain nonlinearities are expressed as:

$$
\left\{\begin{array}{l}
\theta_{\min i} \leq \theta_{i} \leq \theta_{\text {maxi }} \\
\left|\Delta_{i}\right| \leq \delta_{i}
\end{array} \quad i=1,2,\right.
$$

Then, the Eq. (7) can be rewritten as:

$$
\left\{\begin{array}{l}
\dot{x}_{1}=x_{2} \\
\dot{x}_{2}=\frac{A_{1}}{m} \bar{x}_{3}-\theta_{2} x_{2}-\theta_{1}+\Delta_{1} \\
\dot{\bar{x}}_{3}=-f_{1} x_{2}-f_{2} x_{3}+f_{3} x_{4}+f_{4} u_{\mathrm{d}}+\Delta_{2}
\end{array} .\right.
$$

Step1: Define the tracking error as $e_{1}=x_{1}-x_{\mathrm{d}}$, then the time derivative of $e_{1}$ is given by:

$$
\dot{e}_{1}=\dot{x}_{1}-\dot{x}_{\mathrm{d}}=x_{2}-\dot{x}_{\mathrm{d}}
$$

Define the Lyapunov function as $V_{1}=e_{1}^{2} / 2$. The time derivative of $V_{1}$ is given by:

$$
\dot{V}_{1}=e_{1}\left(x_{2}-\dot{x}_{\mathrm{d}}\right) \text {. }
$$

The virtual control input is designed as $x_{2 \mathrm{~d}}=\dot{x}_{\mathrm{d}}-k_{1} e_{1}$ where $k_{1}$ is a positive constant. The second error variable is $e_{2}=x_{2}-x_{2 \mathrm{~d}}$. Thus, Eq. (19) can be rewritten as:

$$
\dot{V}_{1}=e_{1} e_{2}-k_{1} e_{1}^{2} .
$$

Step2: The time derivative of $e_{2}$ is given by

$$
\dot{e}_{2}=\dot{x}_{2}-\dot{x}_{2 \mathrm{~d}}=\frac{A_{1}}{m} \bar{x}_{3}-\theta_{2} x_{2}-\theta_{1}+\Delta_{1}-\dot{x}_{2 \mathrm{~d}} .
$$

The Lyapunov function is defined as:

$$
V_{2}=V_{1}+\frac{1}{2} e_{2}^{2}
$$

Combining Eqs. (20), (21) and (22), the time derivative of $V_{2}$ is obtained as:

$$
\begin{aligned}
\dot{V}_{2}= & e_{2}\left(\frac{A_{1}}{m} \bar{x}_{3}-\widehat{\theta}_{2} x_{2}-\widehat{\theta}_{1}+\Delta_{1}-\dot{x}_{2 d}-\tilde{\theta}_{2} x_{2}-\tilde{\theta}_{1}\right)+ \\
& +e_{1} e_{2}-k_{1} e_{1}^{2},
\end{aligned}
$$

where $\hat{\theta}_{1}$ and $\hat{\theta}_{2}$ are the estimates of $\theta_{1}, \theta_{2}$ respectively, $\tilde{\theta}_{1}$ and $\tilde{\theta}_{2}$ denote the estimation errors. The virtual control is designed as:

$x_{3 \mathrm{~d}}=\frac{m}{A_{1}}\left(-e_{1}+\widehat{\theta_{2}} x_{2}+\widehat{\theta}_{1}+\dot{x}_{2 \mathrm{~d}}-k_{2} e_{2}-\delta_{1} \tanh \left(\frac{e_{2}}{\varepsilon_{1}}\right)\right)$,

where $\tanh (\bullet)$ is the hyperbolic tangent function, which is employed to further attenuate the input chattering, $k_{2}$ is a positive constant.

The third error variable is $e_{3}=\bar{x}_{3}-x_{3 \mathrm{~d}}$ and the Eq. (23) can be obtained as: 


$$
\begin{aligned}
\dot{V}_{2}= & -k_{1} e_{1}^{2}-k_{2} e_{2}^{2}+\frac{A_{1}}{m} e_{2} e_{3}+\Delta_{1} e_{2}- \\
& -\delta_{1} \tanh \left(\frac{e_{2}}{\varepsilon_{1}}\right) e_{2}-e_{2}\left(-\tilde{\theta}_{2} x_{2}-\tilde{\theta}_{1}\right) .
\end{aligned}
$$

Step3: The time derivative of $e_{3}$ is given by:

$$
\dot{e}_{3}=\dot{\bar{x}}_{3}-\dot{x}_{3 \mathrm{~d}}=-f_{1} x_{2}-f_{2} x_{3}+f_{3} x_{4}+f_{4} u_{\mathrm{d}}+\Delta_{2}-\dot{x}_{3 \mathrm{~d}} \text {. }
$$

The Lyapunov function is defined as:

$$
V_{3}=V_{2}+\frac{1}{2} e_{3}^{2}+\frac{1}{2} \rho_{1} \tilde{\theta}_{1}^{2}+\frac{1}{2} \rho_{2} \tilde{\theta}_{2}^{2},
$$

where $\rho_{1}$ and $\rho_{2}$ are the error coefficients.

Combining Eq. (25), (26) and (27), the time derivative of $V_{3}$ is obtained as:

$$
\begin{aligned}
\dot{V}_{3}= & -k_{1} e_{1}^{2}-k_{2} e_{2}^{2}+\frac{A_{1}}{m} e_{2} e_{3}+\Delta_{1} e_{2}-\delta_{1} \tanh \left(\frac{e_{2}}{\varepsilon_{1}}\right) e_{2}+ \\
& +e_{3}\left(-f_{1} x_{2}-f_{2} x_{3}+f_{3} x_{4}+f_{4} u_{\mathrm{d}}+\Delta_{2}-\dot{x}_{3 \mathrm{~d}}\right)- \\
& -e_{2}\left(-\tilde{\theta}_{2} x_{2}-\tilde{\theta}_{1}\right)-\rho_{1} \tilde{\hat{\theta}}_{1} \dot{\hat{\theta}}_{1}-\rho_{2} \tilde{\theta}_{2} \dot{\hat{\theta}}_{2} .
\end{aligned}
$$

The actual control is designed as:

$$
\begin{aligned}
u_{\mathrm{d}} & =\frac{1}{f_{4}}\left(f_{1} x_{2}+f_{2} x_{3}-f_{3} x_{4}+\dot{x}_{3 \mathrm{~d}}-k_{3} e_{3}-\right. \\
& \left.-\delta_{2} \tanh \left(\frac{e_{3}}{\varepsilon_{2}}\right) e_{3}-\frac{A_{1}}{m} e_{2}\right),
\end{aligned}
$$

where $k_{1}$ is a positive constant. The adaptation law is chosen as:

$$
\left\{\begin{array}{l}
\dot{\hat{\theta}}_{1}=-e_{2} / \rho_{1} \\
\dot{\hat{\theta}}_{2}=-e_{2} x_{2} / \rho_{2}
\end{array} .\right.
$$

In case the parameter estimate $\hat{\theta}_{i}$ varies significantly, which may result in a very large input voltage, $\dot{\hat{\theta}}_{i}$ is updated using the following projection type adaption law [16].

$$
\operatorname{Proj}_{\hat{\theta}}\left(\bullet_{i}\right) \begin{cases}0 & \text { if } \hat{\theta}_{i} \geq \theta_{\text {maxi }} \text { and } \bullet_{i}>0 \\ 0 & \text { if } \hat{\theta}_{i} \leq \theta_{\min i} \text { and } \bullet_{i}<0 \quad i=1,2 . \\ \bullet_{i} & \text { otherwise }\end{cases}
$$
yields:

Substituting the Eqs. (29) and (30) into Eq. (28)

$$
\begin{aligned}
\dot{V}_{3} & =-k_{1} e_{1}^{2}-k_{2} e_{2}^{2}-k_{3} e_{3}^{2}+\Delta_{1} e_{2}- \\
& -\delta_{1} \tanh \left(\frac{e_{2}}{\varepsilon_{1}}\right) e_{2} \Delta_{2} e_{3}-\delta_{2} \tanh \left(\frac{e_{3}}{\varepsilon_{2}}\right) e_{3} \leq 0 .
\end{aligned}
$$

Remark2: Because the proposed control strategy is a set point strategy, it is necessary to verify the stability of the system. However, the stability of the internal dynamics of the system is difficult to ensure.
A method in [3], which utilized a new state variable and zero dynamics to validate the stability, can also be used in this study. The extended state variable $x_{5}$ and the dynamic of the pump pressure, which are deduced by Eqs. (12) and (14), respectively, are as follows

$$
\begin{aligned}
& \left\{\begin{array}{ll}
x_{5}=h_{1} \sqrt{\frac{2}{\rho}\left(p_{\mathrm{s}}-p_{1}\right)}-h_{2} \sqrt{\frac{2}{\rho}\left(p_{2}-p_{\mathrm{t}}\right)} & \dot{x}_{1} \geq 0 \\
x_{5}=h_{1} \sqrt{\frac{2}{\rho}\left(p_{1}-p_{\mathrm{t}}\right)}-h_{2} \sqrt{\frac{2}{\rho}\left(p_{\mathrm{s}}-p_{2}\right)} & \dot{x}_{1}<0
\end{array},\right. \\
& \left\{\begin{array}{l}
\dot{p}_{\mathrm{s}}=\dot{p}_{1}+\frac{\rho A_{1}^{2} \dot{x}_{\mathrm{p}} \ddot{x}_{\mathrm{p}}}{k_{\mathrm{q}}^{2} k_{\mathrm{x}}^{2} \alpha^{2} u_{\max }^{2}}+\lambda \frac{l}{d} \frac{\rho \ddot{x}_{\mathrm{p}}^{2}}{2}+\sigma_{1} \dot{p}_{1} \\
\dot{p}_{\mathrm{s}}=\dot{p}_{2}+\frac{\rho A_{2}^{2} \dot{x}_{\mathrm{p}} \ddot{x}_{\mathrm{p}}}{k_{\mathrm{q}}^{2} k_{\mathrm{x}}^{2} \alpha^{2} u_{\max }^{2}}+\lambda \frac{l}{d} \frac{\rho \ddot{x}_{\mathrm{p}}^{2}}{2}+\sigma_{2} \dot{p}_{2}
\end{array}\right.
\end{aligned}
$$

Case1. Zero output: In this case, the output of the system should be kept at zero. This means that $x_{\mathrm{d}}=\dot{x}_{\mathrm{d}}=\ddot{x}_{\mathrm{d}}=\dddot{x}_{\mathrm{d}}=x_{1}=\dot{x}_{1}=\ddot{x}_{1}=\dddot{x}_{1}=0$. Thus, the control input approaches zero in this case according to Eq. (29). Accordingly, the flows of the two chambers are also near zero. Neglecting the leakage, we can get $\dot{p}_{1}=\dot{p}_{2}=0$ from Eq. (3). Thus, the chamber pressure will be regulated at constant values. Consequently, the pump pressure is also a constant value and $\dot{p}_{\mathrm{s}}=0$ according to Eqs. (12), (14), and (34). As a result, $x_{5}$ will tend to be a constant value and $\dot{x}_{5}=0$.

Case 2. Constant output: In this case, $x_{\mathrm{d}}=x_{1} \neq 0$, $\dot{x}_{\mathrm{d}}=\ddot{x}_{\mathrm{d}}=\dddot{x}_{\mathrm{d}}=\dot{x}_{1}=\ddot{x}_{1}=\dddot{x}_{1}=0$. Based on Eqs. (29) and (34), the control input and $\dot{p}_{\mathrm{s}}$ will approach zero again, and the rest of the analysis will be the same as Case1, which means that in a set point strategy, the internal dynamics of the system will be stable.

\section{EXPERIMENTAL RESULTS}

The setup is presented in Fig. 3. The position of the cylinder is measured by a displacement sensor (LS 628C). The pressures in the two cylinder chambers and supply pressure are measured by pressure sensors (HDP702). A dSpace1104 data acquisition data board is used to acquire the feedback signals from sensors and to generate control signals. The variable pump is a Danfoss load-sensing pump. The parameters of the system are shown in Table 1.

Fig. 4 depicts the harmonic reference tracking test for the proposed method by selecting $\alpha$ as 0.86 . It can be observed that the actual trajectory follows the desired position well. The pump pressure and chamber pressures are shown in Fig. 5, in which the maximum pressure margin is 7.5 bar. It is less than the 
preset pressure margin 14 bar in the pump. Moreover, with the increase in the flow, the pressure margin is boosted, and the decrease of the pressure margin is associated with decreases in flow. A higher pressure margin always appears at the middle stroke of the cylinder in both forward and backward motion, which could verify the effectiveness of the energy-saving method.

Table 1. Parameters of the system

\begin{tabular}{|c|c|c|c|}
\hline $\begin{array}{l}\text { Total leakage } \\
\text { coefficient }\end{array}$ & $3 \times 10^{-11}$ & Oil bulk modulus & $1 \times 10^{9} \mathrm{~Pa}$ \\
\hline $\begin{array}{l}\text { Piston/rod } \\
\text { diameter }\end{array}$ & $40 / 30 \mathrm{~mm}$ & $\begin{array}{l}\text { equivalent mass } \\
\text { of the load }\end{array}$ & $15 \mathrm{~kg}$ \\
\hline Oil density & $890 \mathrm{~kg} / \mathrm{m}^{3}$ & Viscous damping & $800 \mathrm{~N} / \mathrm{m}$ \\
\hline$k_{\mathrm{q}} k_{\mathrm{x}}$ & $8.8 \times 10^{-7}$ & $\delta_{1}$ & 0.005 \\
\hline$\delta_{2}$ & 1 & $\sigma_{1}$ & 0.2 \\
\hline$\sigma_{2}$ & 0.05 & $k_{1}$ & 100 \\
\hline$k_{2}$ & 200 & $k_{3}$ & 135 \\
\hline
\end{tabular}

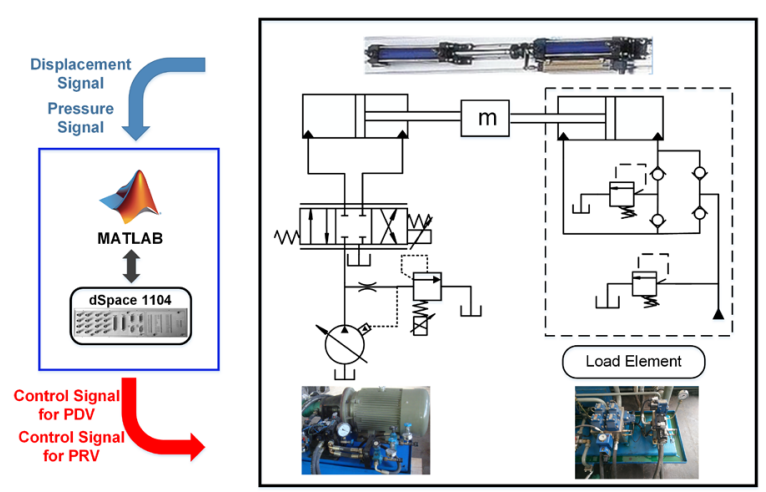

Fig. 3. Schematic of the equipment

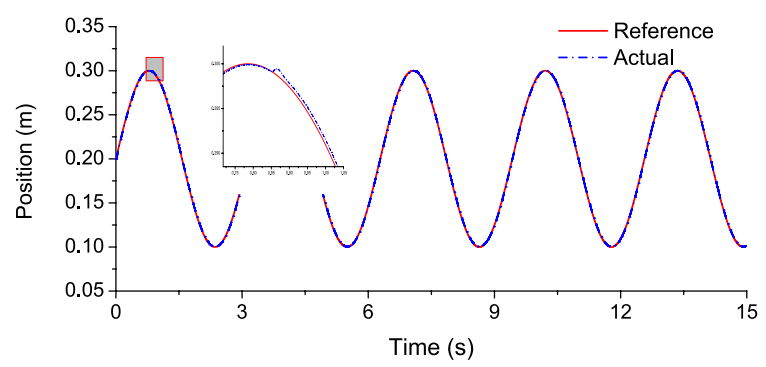

Fig. 4. Tracking performance

The parameter estimates are shown in Fig. 6, and the tracking errors of three different methods are shown in Fig. 7. It can be observed that the proposed method exhibits a better tracking performance than PID control with a maximum tracking error of 1.2 $\mathrm{mm}$. If sliding mode control is used to achieve the same level of tracking performance, high feedback gain must be employed, which may result in overshoots due to the low-pressure difference and the chattering problem. As can be seen from Fig. 7, a significant tracking error of $3 \mathrm{~mm}$ occurs in the peaks of the reference signal. Moreover, the average tracking errors of the proposed method and the sliding mode controller are $0.75 \mathrm{~mm}$ and $0.9 \mathrm{~mm}$, respectively. Hence, the proposed method has a better tracking

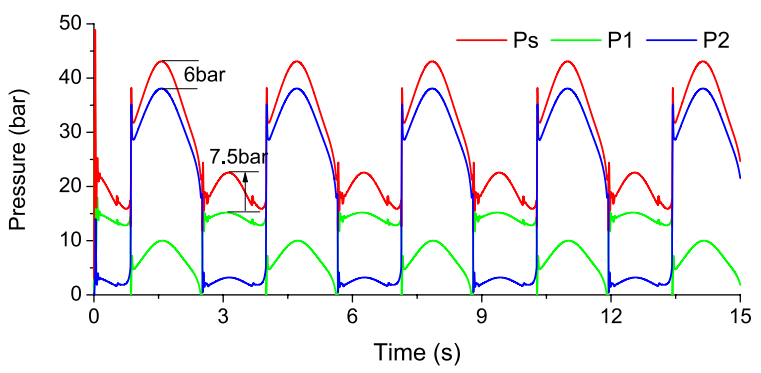

Fig. 5. Pump pressure and cylinder chamber pressures

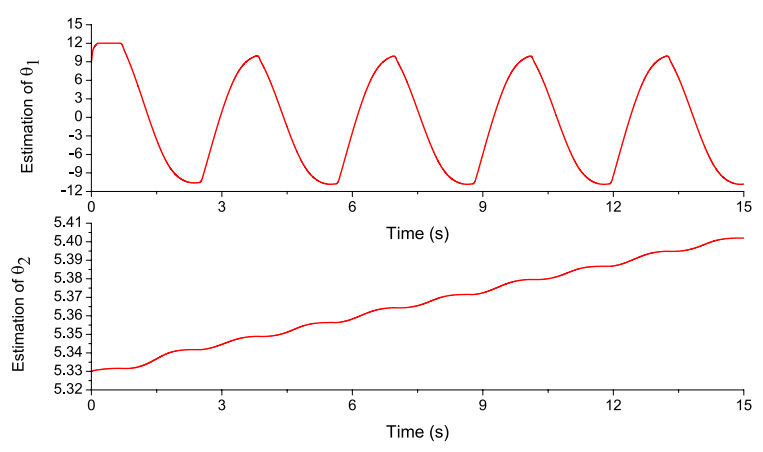

Fig. 6. Estimation results

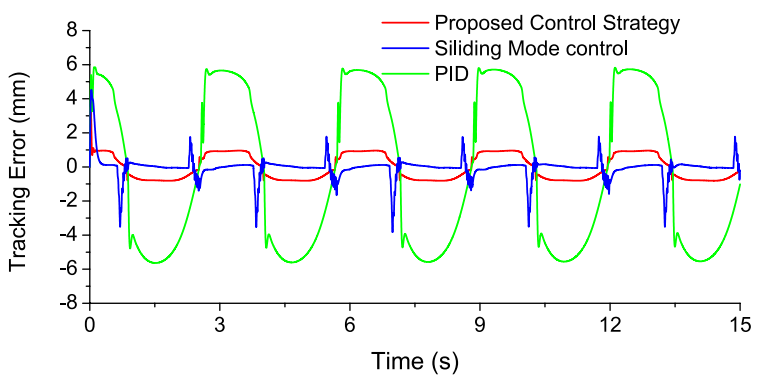

Fig. 7. Tracking errors $(\alpha=0.86)$

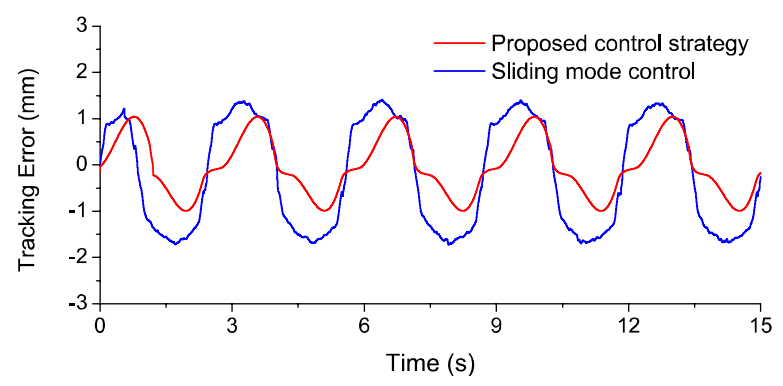

Fig. 8. Tracking errors $(\alpha=0.7)$ 
performance than the sliding mode control regarding the average error and maximum error. To further verify the tracking performance of the proposed method, another experiment was carried out by selecting the $\alpha$ as 0.7 . As can be seen from Fig. 8, the proposed method demonstrates a better tracking performance compared with the sliding mode controller. It can also be found that although the tracking error of the proposed method is slightly smaller when $\alpha$ is 0.7 , it is not obvious. Meanwhile, the energy-saving performance will degrade by choosing a smaller value of $\alpha$. The suitable value of $\alpha$ is determined by trialand-error and eventually chosen as 0.86 by taking both energy saving and position tracking performance into account.

To evaluate the energy-saving performance of the proposed method, it is compared with a fixed displacement system. The system pressure of the fixed displacement system is assumed to be 50 bar, and the flow provided by the pump is assumed to be $16 \mathrm{l} / \mathrm{min}$, which is slightly higher than the maximum demand flow. The energy-saving performance of the proposed system was also evaluated by comparing the energy efficiency with that of the fixed displacement system with VSPC, which is similar to the existing VSPC system. Neglecting the energy loss across the throttle valve, the supply energy of the three methods are shown in Fig. 9. The energy is calculated by $p_{\mathrm{s}} Q_{\mathrm{L}}$, in which $Q_{\mathrm{L}}$ is the flow rate of the pump. It can be observed from Fig. 9 that the supply energy of a fixed displacement system is $20 \mathrm{~kJ}$, whereas the supply energies are $14.8 \mathrm{~kJ}$ and $7.5 \mathrm{~kJ}$ in the fixed displacement system with VSPC and the proposed system, respectively. Thus, it can be concluded that the proposed method is capable of saving $62.5 \%$ energy in comparison with a fixed displacement system and approximately $49 \%$ energy in comparison with a fixed displacement system with VSPC.

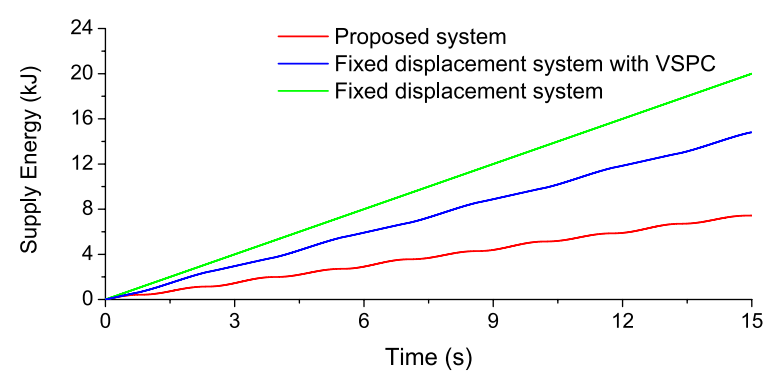

Fig. 9. Supply energy of the system

To further verify the proposed method, multistep reference tests were conducted by selecting $\alpha$ as 0.82 . The results are depicted by Figs. 10 to 12. It can be observed from Fig. 10 that the proposed method succeeded in realizing position tracking and the dynamic behaviour of the presented system is good. The pump pressure and chamber pressures are shown in Fig. 11. It can be observed that the maximum pressure margin is 10 bar and the pump pressure remains at 14 bar when the desired pump pressure is lower than the preset pressure margin. The supply energy during the whole motion cycle is shown in Fig. 12. The system pressure of the fixed displacement system is assumed to be 45 bar. It is shown in Fig. 12 that the supply energy of the fixed displacement system is $28 \mathrm{~kJ}$. Compared with the fixed displacement system, the fixed displacement system with VSPC saved approximately $62 \%$ energy, and the proposed system saved approximately $90 \%$ energy.

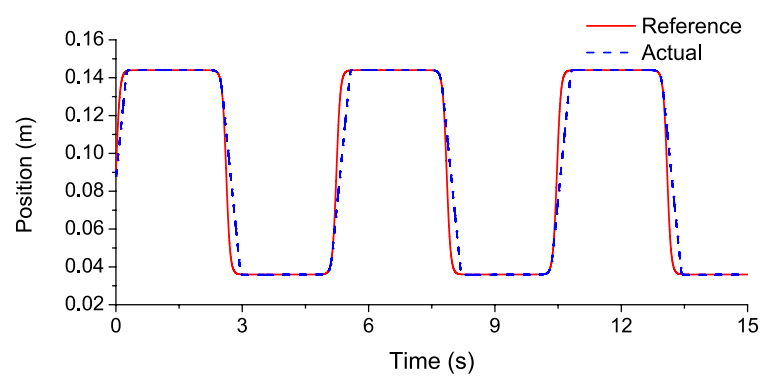

Fig. 10. Tracking performance

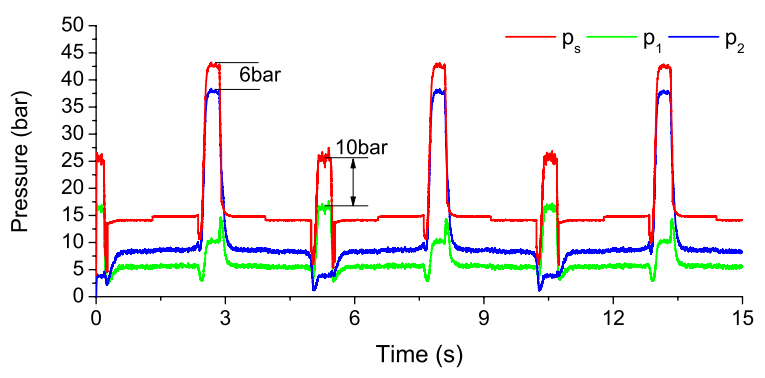

Fig. 11. Pump pressure and cylinder chamber pressures

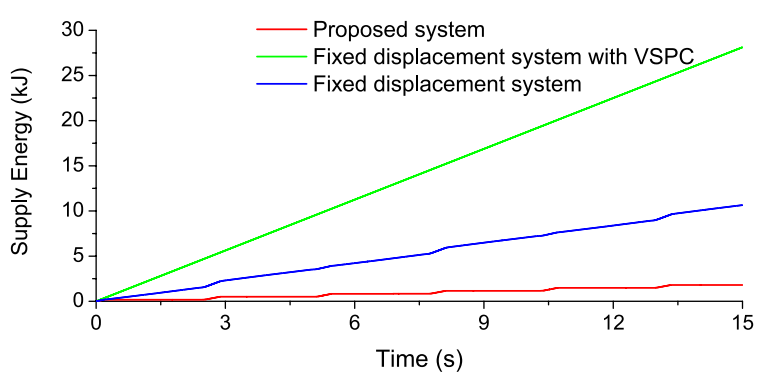

Fig. 12. Supply energy of the system

\section{CONCLUSIONS}

1) A VSPC based load-sensing structure is presented in order to reduce the overflow loss and excess 
pressure loss across the PDV. Thus, the flow and pressure of the EHSS can both meet the control demand. Additionally, an adaptive backstepping sliding mode control is introduced for position tracking.

2) Compared with the fixed displacement system, the proposed method is capable of saving $62.5 \%$ and $90 \%$ of supply energy in harmonic reference tests and multi-step reference tests, respectively. The results also demonstrate that the presented method has a better energy-saving performance in comparison with [3] and [4] because the overflow loss can be reduced in this study.

3) The proposed method exhibits a good tracking capacity with a maximum tracking error of 1.2 $\mathrm{mm}$ in the harmonic reference tests. Furthermore, the tracking performance and dynamic behaviour for the multi-step reference tests are also good.

This method could also be combined with the independent metering to save even more energy in mobile machinery and other applications because the load-sensing pump presented here is widely used in engineering machinery.

\section{ACKNOWLEDGEMENTS}

The project is funded in part by the Priority Academic Program Development of Jiangsu Higher Education Institutions (PAPD), the 2016 annual general university graduate research and innovation program of Jiangsu Province, China (KYLX16 0525). We also give our great thanks to Keivan Baghestan, Amirkabir University of Technology, Tehran, Iran, for his valuable suggestions.

\section{REFERENCES}

[1] Lu, X.C., Chen, Q.B., Zhang, Z.J. (2014). The electric vehicle routing optimizing algorithm and the charging stations' layout analysis in Beijing. International Journal of Simulation Modelling, no. 13, no. 1, p. 116-127, D0l:10.2507/ IJSIMM13(1)CO4.

[2] Casoli, P., Pompini, N., Riccò, L. (2015). Simulation of an excavator hydraulic system using nonlinear mathematical models. Strojniški vestnik - Journal of Mechanical Engineering, vol. 61, no. 10, p. 583-593, D0l:10.5545/sv-jme.2015.2570.

[3] Baghestan, K., Rezaei, S.M., Talebi, H.A., Zareinejad, M. (2015). An energy-saving nonlinear position control strategy for electro-hydraulic servo systems. ISA Transactions, vol. 59, p. 268-279, D0I:10.1016/j.isatra.2015.10.012.

[4] Tivay, A., Zareinejad, M., Rezaei, S. M., Baghestan, K. (2014). A switched energy saving position controller for variablepressure electro-hydraulic servo systems. ISA Transactions, vol. 53, p. 1297-1306, D0I:10.1016/j.isatra.2014.04.010.
[5] Guo, K., Wei, J., Fang, J., Feng, R., Wang, X. (2015). Position tracking control of electro-hydraulic single-rod actuator based on an extended disturbance observer. Mechatronics, vol. 27, no. 4, p. 47-56, D0l:10.1016/j.mechatronics.2015.02.003.

[6] Mohanty, A., Yao, B. (2011). Indirect adaptive robust control of hydraulic manipulators with accurate parameter estimates. IEEE Transactions on Control Systems Technology, vol. 19, no. 3. p. 567-575, D0I:10.1109/TCST.2010.2048569.

[7] Mohanty, A., Yao, B. (2011). Integrated direct/indirect adaptive robust control of hydraulic manipulators with valve deadband. IEEE/ASME Transactions on Mechatronics, vol. 16, no. 4. p. 707-715, DOI:10.1109/TMECH.2010.2051037.

[8] Micheal, J., Rahmat, M.F., Abdul, N., Lai, W.K. (2013). Feed forward linear quadratic controller design for an industrial electro hydraulic actuator system with servo valve. International Journal on Smart Sensing and Intelligent Systems, vol. 6, no. 1, p. 154-170.

[9] Niksefat, N., Sepehri, N. (2001). Designing robust force control of hydraulic actuators despite system and environmental uncertainties. IEEE Control Systems, vol. 21, no. 2, p. 66-77, D0l:10.1109/37.918266.

[10] Gao, B., Shao, J., Yang, X. (2014). A compound control strategy combining velocity compensation with ADRC of electrohydraulic position servo control system. ISA Transactions, vol. 53, no. 6, p. 1910-1918, D0I:10.1016/j.isatra.2014.06.011.

[11] Chiang, M.H., Yeh, Y.P., Yang, F.L., Chen, Y.N. (2005). Integrated control of clamping force and energy-saving in hydraulic injection moulding machines using decoupling fuzzy sliding-mode control. The International Journal of Advanced Manufacturing Technology, vol. 27, no. 1, p. 53-62, DOI:10.1007/s00170-004-2138-z.

[12] Cho, S.H., Noskievič, P. (2012). Position tracking control with load-sensing for energy-saving valve-controlled cylinder system. Journal of Mechanical Science and Technology, vol. 26, no. 2, p. 617-625, Dol:10.1007/s12206-011-1032-5.

[13] Lovrec, D., Kastrevc, M., Ulaga, S. (2009). Electro-hydraulic load sensing with a speed-controlled hydraulic supply system on forming-machines. The International Journal of Advanced Manufacturing Technology, vol. 41, no. 11, p. 1066-1075, D0I:10.1007/s00170-008-1553-y.

[14] Axin, M., Eriksson, B., Krus, P. (2014). Flow versus pressure control of pumps in mobile hydraulic systems. Journal of Systems and Control Engineering, vol. 228, no. 4, p. 245-256, DOI:10.1177/0959651813512820.

[15] Minav, T., Hänninen, H., Sinkkonen, A., Laurila, L., Pyrhönen, J. (2014). Electric or hydraulic energy recovery systems in a reach truck - a comparison. Strojniški vestnik - Journal of Mechanical Engineering, vol. 60, no. 4, p. 232-240. DOI:10.5545/sv-jme.2013.1581.

[16] Lu, L., Yao, B. (2014). Energy saving control of a hydraulic manipulator using five cartridge valves and one accumulator. IEEE Transactions on Industrial Electronics, vol. 61, no. 12, p. 7046-7054, D0I:10.1109/TIE.2014.2314054.

[17] Kovari, A. (2015). Effect of leakage in electrohydraulic servo systems based on complex nonlinear mathematical model and experimental results. Acta Polytechnica Hungarica, vol. 12, no. 3, p. 129-146, D0l:10.12700/APH.12.3.2015.3.8. 